\title{
Ailevi Akdeniz Ateşi Olan 40 Gebenin Perinatal Sonuçları
}

\author{
Perinatal Outcomes of 40 Pregnancies with Familial Mediterranean Fever
}

\author{
Ebru Alıı DAVUTOĞLU ${ }^{1}$, Ayşegül ÖZEL ${ }^{1}$, Hakan ERENEL ${ }^{1}$, Rıza MADAZLI ${ }^{1}$, \\ 1. İstanbul Üniversitesi, Cerrahpaşa Tıp Fakültesi, Kadın Hastalıkları ve Doğ., A.B.D., Perinatoloji Bilim Dalı, İstanbul
}

\section{$\ddot{O Z Z E T}$}

Amaç: Ailevi Akdeniz Ateşi (AAA) tanısı olan, gebe olguların maternal ve fetal sonuçlarının değerlendirilmesi amaçlanmıştır.

Gereç ve Yöntemler: Çalıșmamız 2013-2017 yılları arasında üniversitemizin perinataloji kliniğinde gebelikleri takip edilmiş 40 AAA olgusunun dosya verilerinin retrospektif olarak incelenmesiyle yapıldı. Hastaların klinik özellikleri, obstetrik ve perinatal sonuçları değerlendirildi.

Bulgular: Ortalama hasta yaşı $30.75 \pm 6.15$, nulliparite oranı $\% 42,5$ bulundu. Ortalama hastalık süresi 8,36 \pm 5.44 yll olarak bulundu. Gebelik sirasinda 7 hasta atak geçirdi. Hastaların \%90' ' medikal tedavi almaktaydı. Ortalama doğum haftası $37,3 \pm 4.5$, ortalama doğum kilosu $3115 \pm 547 \mathrm{gr}$ bulundu. Preterm doğum, fetal büyüme klsitlllı̆̆ı,preeklampsi görülme oranlarl sirasiyla; \%10, \%5, \%10, olarak bulundu. Fetal ve neonatal kayıp, ile konjenital anomalili doğum gözlenmedi.

Sonuç: AAA tanıl gebelik olgularının yönetiminde multidisipliner yaklaşım ile takip ve tedavinin sağlanması olumlu maternal ve fetal sonuçlar ile ilişkilidir.

Anahtar Kelimeler: ailevi akdeniz ateşi, perinatal sonuçlar

ABSTRACT

Objective: To evaluate maternal and fetal outcomes of pregnancies diagnosed with Familial Mediterranean Fever (FMF).

Material and Methods: Retrospective analysis of 40 pregnancies diagnosed with FMF who received antenatal care at our perinatology unit between 2012-2017 included in this clinical study. Clinical characteristics of patients, perinatal and maternal outcomes were evaluated.

Results: The mean patient age was $30.75 \pm 6.15$ and the nulliparity rate was $42,5 \%$. The mean duration of illness was found to be $8,36 \pm 5.44$ years. 7 patients had an attack during pregnancy. $90 \%$ of the patients received medical treatment. Mean gestational age at delivery was $37,3 \pm 4.5$, mean birth weight was $3115 \pm 547 \mathrm{~g}$. Preterm delivery, fetal growth restriction and preeclampsia rates were 10, 5, 10 \%respectively. Fetal and neonatal loss, congenital anomalous birth was not observed.

Conclusion: Multidisciplinary approach to the care of pregnancy diagnosed with FMF is associated with good maternal and fetal outcomes.

Keywords: familial mediterranean fever, perinatal outcome

İletişim Bilgileri

Sorumlu Yazar: Ayşegül ÖZEL

Yazışma Adresi: İstanbul Üniversitesi Cerrahpaşa Tıp Fakültesi Kadın Hastalıkları ve Doğum Ana Bilim Dalı, Perinatoloji Bilim Dalı Fatih, İstanbul

E-posta: ozelaysegul@hotmail.com

Tel: +90 (532) 5086353

Makale Geliş Tarihi: 07.08..2017

Makale Kabul Tarihi: 06.09.2017

DOI: http://dx.doi.org/10.16948/zktipb.333212

\section{GİRIŞ}

AAA (Ailevi Akdeniz Ateşi), periyodik ateş ataklar1, aseptik serozit ve sinovit ile seyreden, otozomal resesif geçişli bir hastalıktır (1).

Hastaların çoğu 20 yaşından önce tanı alır (2). Olguların \%95' inde karın ağrısı hastalı̆̆ın ilk belirtisi olarak bildirilmektedir. Ağrı batın alt kadranlarından başlayıp tüm abdomene yayılır.

Ayırıcı tanıda appendisit, safra kesesi hastalıkları, akut pankreatit, porfiria, kronik inflamatuar barsak hastalıkları ve jinekolojik patolojiler (over kist torsiyonu, korpus luteum kist rüptürü, ektopik gebelik1,endometriozis) akla gelmelidir $(3,4)$. AAA ' nin Türkiye ' deki prevelansı 1/400-1/1000 olarak tahmin edilmektedir $(5,6)$.

Hastalık tüm serozal membranları (plevra, perikard, periton) tutabilir. Kronik rekürren serosal inflamasyon sonucu gelişen peritoneal yapışıklıklara bağ$l_{1}$ infertilite ve amiloidoz en önemli uzun dönem komplikasyonlarıdır (3). Amiloidoza bağlı renal yetmezlik, AAA olan hastalarda mortalitenin en önemli nedenidir $(2,8,12)$. Kolşisin, AA ataklarını ve şiddetini azaltan önemli bir ilaçtır $(7,8)$.

Yapılan çalışmalar, Kolşisin tedavisi ile hastaların \%60-75 'inde tam remisyon sağlandığını, ancak \%5-10' unun tedaviye yanıtsız olduğunu göstermiştir (8). 1970 'li y1llarda kromozomal anomalilere yol açabileceği endişesiyle, gebe kalmadan 3 ay öncesinde tedaviye ara verildi (8).

Günümüzde, ilaca ara verilmesi halinde semptomların alevlendiği bilindiğinden, ilaca devam edilmesi önerilir ve çalıșmalar ilacın gebelikte kullanımının güvenli olduğunu göstermiştir $(7,10)$.

$\mathrm{Bu}$ gebelere, fetal karyotip analizi amaciyla rutin amniyosentez önerilmesi konusunda görüşler farklılık gösterir $(3,4,9)$. Ancak, kolşisin ile tedavi edilen ve edilmeyen hasta grubu ile yapilan bir çalışmada malformasyon oranlarında fark olmadığ 1 ortaya konmuştur (11).

AAA olan gebelerde hastalığın seyri değișkendir. Bazı hastalar remisyona girerken bazılarında atak sıklığ $\operatorname{artar}$ (3). Bu konuda yeterli çalışma olmasa da, gebeliğin, AAA ataklarının süresini ve sıklığını değiștirmediği kabul edilir. Aksine, tekrarlayan atakların gebelik komplikasyonlarını artırdığına inanılır. 
Mevcut çalışmanın amacı, Türkiye'deki 3. basamak bir sağlık merkezinin AAA ile komplike olan gebeliklerinin maternal ve fetal sonuçlarının verilerini retrospektif olarak incelemektir.

\section{GEREÇ ve YÖNTEM}

2013 ve 2017 yılları arasında üniversitemizin romatoloji kliniğinde takip edilen, AAA olan, 40 gebe olgunun perinatoloji kliniğimizdeki dosya kayitları retrospektif olarak araştırıldı.

Hastaların gebelikleri boyunca hem Perinatoloji hem de Romatoloji uzmanı tarafindan takipleri gerçekleştirildi. Antenatal takip ve tıbbi kayıtlar anne ve yenidoğan için değerlendirildi. İlk antenatal muayenede temel parametreler kaydedildi.

Maternal yaş, parite, hastalığın süresi, gebelikte atak varlığı ve ilaç kullanımına ilişkin kayıtlar alındı.

Maternal sonuçlar doğumdaki gestasyonel yaş, doğum şekli, preeklampsi ve gebelikte atak öyküsü, 22. gebelik haftasındaki ortalama uterin arter doppler PI değeri ;perinatal sonuçlar; prematürite (37 haftadan önce doğum), fetal büyüme kısıtl11ığ (FBK; tahmini fetal ağırlık $\leq 10$. persentil), ölü doğum (intrauterin ölüm $\geq 20$. gestasyonel hafta), neonatal ölüm (doğum sonrası ilk 28 gündeki ölüm), doğum ağırlığ 1 ve konjenital anomali verilerini içeren kayıtlar tüm olgularda değerlendirildi.

\section{BULGULAR}

Olguların klinik karakteristikleri Tablo 1' de özetlenmiştir. Ortalama yaş $30.75 \pm 6.15$ idi.

Hastaların \%42.5'i nullipar gebelerden oluşmaktayd1. Hastalarda AAA tanı süresi, ortalama 8,36 \pm 5.44 y1l idi.

Hastaların 22. gebelik haftasinda ortalama uterin arter PI değeri $0.91 \pm 0.24$ idi. Olguların \% 17.5 ' i gebelik sırasında atak geçirdi. Hastaların tümünün atak sırasında ilaca kendilerinin ara vermiş olduğu saptandı. Hastaların hiçbirinde uzun dönem komplikasyonu olarak amiloidoz saptanmadi.

Gebelerin\%90'1medikaltedavialmaktaydı(Tablo1). Tedavide kullanılan ana ilaç kolşisin iken 2 hastaya kortikosterid, 2 hastaya da Anakinra (IL1 reseptör antagonisti) ile kombine tedavi başlanmıştı.

Obstetrik ve perinatal sonuçlar Tablo 2' de özetlendi. Ortalama doğum haftası $37,3 \pm 4.5$, ortalama doğum kilosu $3115 \pm 547$ gr idi. Sezeryan ile doğum yapan 22 hastada (\%55) primer sezeryan oran1 \%57.2 idi. 34 'üncü gebelik haftasindan önce 1 hasta doğum yaparken (\%2.5), 37 haftadan önce doğum yapan 4 (\%10), fetal büyüme kısıtlılı̆̆ olan 4 (\%10) preeklampsi gelişen $2(\% 5)$ olgu izlendi. 1 olguda $(\% 2.5)$ gebeliğin 10. haftasında abortus oldu.

Fetal, neonatal kayıp ve konjenital anomali doğum görülmedi.
Tablo 1: Hastaların klinik özellikleri.

\begin{tabular}{|l|l|}
\hline \multicolumn{1}{|c|}{$\mathbf{N}$} & \multicolumn{1}{|c|}{40} \\
\hline Yaş (yıl, ortalama \pm SD) & $30,75 \pm 6.15$ \\
\hline Nulliparite (n, \%) & $17,42.5$ \\
\hline Hastalık süresi (yıl, ortalama \pm SD) & $8,36 \pm 5.44$ \\
\hline Uterin arter doppler PI (ortalama \pm SD) & $0.91 \pm 0.24$ \\
\hline Gebelikte atak geçiren hasta (n,\%) & $7,17.5$ \\
\hline \multicolumn{1}{|c|}{ Medikal tedavi } & 32,80 \\
\hline Kolşisin (n, \%) & 2,5 \\
\hline Kolşisin + Kortikosteroid (n, \%) & 2,5 \\
\hline Kolşisin + Anakinra (n, \%) & \\
\hline
\end{tabular}

Tablo 2: Obstetrik ve perinatal sonuçlar.
\begin{tabular}{|l|l|}
\hline Ortalama doğum haftası & $37,3 \pm 4.5$ \\
\hline Ortalama doğum kilosu (gr) & $3115 \pm 547$ \\
\hline Sezeryan ile doğum (n,\%) & 22,55 \\
\hline Geçirilmiş sezeryan (n,\%) & $10,45.5$ \\
\hline Primer sezeryan (n,\%) & $12,54.5$ \\
\hline$\leq 34$ haftada doğum (n,\%) & $1,2.5$ \\
\hline$<37$ haftada doğum (n,\%) & 4,10 \\
\hline Preeklampsi (n,\%) & 2,5 \\
\hline Fetal büyüme kısıtlı̆̆ı $(n, \%)$ & 4,10 \\
\hline Düşük (n,\%) & $1,2.5$ \\
\hline Fetal kayıp (n,\%) & 0,0 \\
\hline Neonatal kayıp (n,\%) & 0,0 \\
\hline Konjenital anomali doğum (n,\%) & 0,0 \\
\hline
\end{tabular}

\section{TARTIŞMA}

Tekrarlayan poliserozit olarak da bilinen AAA, aile içinde ve sıklıkla Akdeniz çevresinde yaşayan etnik grupları (Yahudi, Türk, Ermeni, Arap) etkileyen, otozomal resesif geçişli bir hastalıktır $(1,2,5)$. Gebelik seyri ve sonuçları AAA olgularında farklıl1k gösterebilir. AAA' de tekrarlayan ateş ve serozit atakları, düşük ve erken doğum oranlarının artışıyla ilişkilendirilmiştir $(3,4,11)$.

Bu konuyla ilgili kısıtlı vaka serileri yanında Ofir ve arkadaşlarının yaptığı 239 olgudan oluşan çalışma literatürde dikkat çekmektedir. Bu çalışmada AAA' nin tekrarlayan düşük ve erken doğum için bağımsız bir risk faktörü olduğu, bu hastalarda yardımc1 üreme teknikleriyle gebe kalma oranının normal popülasyondan daha fazla olduğu vurgulanmıştır (13).

Yasar ve arkadaşlarının yaptığı, 46 gebe olgudan oluşan çalışma bu popülasyonda tekrarlayan düşük oranının anlamlı şekilde fazla olduğunu ortaya koymaktadır (14). Ben-Cherit bu çalışmaların aksine AAA' nin, erken ve geç dönem düşükleriyle ilişkisinin olmadığını göstermiştir (15). Çalışmamız tek bir merkezin kısıtlı sayıdaki olgularını analiz etmekte ve bunların tamamı spontan gebeliklerden oluşmaktadır. Hastaların hiçbirinde tekrarlayan düşük öyküsü bulunmamaktadır. Ortalama doğum haftas 137,3 $\pm 4.5,37$. haftadan önce doğum oran $1 \% 10$ 'dur. 
Çalışmalar AAA olan gebelerde sezeryan ile doğum oranının yüksek olduğunu vurgular yönde olsa da $(11,14)$, Ofir ve arkadaşları AAA' nin sezeryan için bağımsız bir risk faktörü olmadığını ortaya koymuştur (13). Hasta grubumuzda sezeryan ile doğum oranının literatürdeki benzer çalışmalarlardan daha yüksek olduğu gözlenmiştir. Özellikle primer sezeryan oran1 \%54,5 ile bu grupta beklenmedik şekilde yüksek bulunmuştur. Hastanın doğum şeklindeki tercihi bu durumu açıklayabilir.

Hasta grubumuzun \%90’1 atakların önlenmesi için ilaç kullanmaktadır. Bu oran Türkiye 'de Yaşar ve arkadaşlarının yaptığı çalışmadaki orandan daha yüksektir (14). Yine bu çalışmadan farklı olarak hastalardan 2'si steroid 2'si de Anakinra ile kolşisin kombine tedavisini kullanmaktadır. Transplasental geçişi olan kolşisinin, hücre bölünmesini etkileyip kromozomal ve yapısal bozukluklara yol açabileceği ileri sürülmüş ancak pek çok çalışma gebelikte kullanımının güvenli olduğunu ortaya koymuştur $(11,16-18)$.

Son y1llarda literatürde, AAA olan gebelerin tedavisinde IL 1 reseptör antagonisti, Anakinra kullanımı ile ilgili olgu sunumları yer almaktadır.

Çalışmalar, bu ilaçla gebelikte atakların kontrolünün güvenle yapılabildiğini, fetal gelişim ve anomali sıklığında artış olmadığını vurgulamaktadır $(19,20)$. Çalışmamızın da desteklediği gibi AAA olan gebelerin perinatal sonuçlarının genel popülasyona benzer olduğu kabul edililebilir (4, $13,14)$. AAA tanılı gebelik olgularının yönetiminde multidisipliner yaklaşım ile takip ve tedavinin sağlanması olumlu maternal ve fetal sonuçlar ile ilişkilidir.

Çalışmamızın en önemli kısıtlılığı vaka sayısının sınırlı olması ve kontrol grubunu içermemesidir.

\section{KA Y N A KLAR}

1. Dormer AE, Hale JF. Familial Mediterranean fever: a cause of periodic fever Br. Med J. 1962 Jan ; 13 (1):87-9

2. Ben-Chetrit E, Levy M. Familial Mediterranean fever. Lancet $1998 \mathrm{Feb} ; 28$ (351):659-64

3. Ben-Chetrit E, Levy M. Reproductive system in Familial Mediterranean fever: an overview. Ann Rheum Dis 2003 Oct ; 62 (10) :916-9.

4. Mijatovic V, Hompes PG, Wouters MG. Familial Mediterranean fever and its implications for fertility and pregnancy. Eur J Obstet Gynecol Reprod Biol 2003 Jun ; 10 (108):171-6

5. Tunca M, Akar S, Onen F, Ozdogan H, Kasapcopur O, Yalcinkaya $F$ et al. Familial Mediterranean fever) in Turkey: results of a nationwide multicenter study. Medicine 2005 Jan; 84 (1):1-11.

6. Cobankara V, Fidan G, Turk T, Zencir M, Colakoglu M, Ozen S The prevalence of familial Mediterranean fever in the Turkish province of Denizli: a field study with a zero patient design. Clin Exp Rheumatol 2004 Jul-Aug; 22 (4): 27-30.

7. Michael O, Goldman RD, Koren G. Safety of colchicine therapy during pregnancy. Can Fam Physician 2003 Aug; 49 :967-9.

8. Ben chetrit E, Levy M, Colchicine: 1998 update. Semin Arthritis Rheum 1998 Aug ;28 (1):48-59.

9. Berkenstadt M, Weisz B, Cuckle H, Di-Castro M, Guetta E, Barski G. Chromosomal abnormalities and birth defects among couples with colchicine treated Familial Mediterranean fever. Am J Obstet Gynecol 2005 Oct ;193 (4):1513-6

10. Ditkoff EC,Sauer MV.Successful pregnancy in a Familial Mediterranean fever patient following assisted reproduction. J Assist Reprod Genet 1996 Sep ;13 (8):684- 5.

11. Rabinovitch O, Zemer D, Kukua E, Sohar E, Mashiach S.Colchicine treatment in conception and pregnancy: 231 pregnancies with Familial Mediterranean fever. Am J Repord Immunol 1992 Oct-Dec; $28(3-4): 245-6$

12. Zemer D, Pras M, Sohar E, Modan M,Cabili S, Gafni J. Colchicine in the prevention and treatment of the amyloidosis of familial Mediterranean fever. $N$ Engl J Med 1986 Apr ;314 (16):1001-3.

13. Ofir D, Levy A, Wiznitzer A, Mazor M, Sheiner E Familial Mediterranean fever : An independent risk factor for preterm delivery European Journal of Obstetrics \& Gynecology and Reproductive Biology 2008 Dec;141 (2) :115-118

14. Yasar 0, Iskender C, Kaymak O, Yaman TS, Uygur D, Danıman $N$ Retrospective evaluation of pregnancy outcomes in women with familial Mediterranean fever J Matern Fetal Neonatal Med 2014 May; 27(7): 733-736

15. Ben-Cherit E, Ben-Cherit A, Berkun Y, Ben-Cherit E Pregnancy outcomes in women with familial Mediterranean fever receiving colchicine: is amniocentesis justified? Arthritis Care Res 2010 Feb; 62(2): $143-148$

16. Ehrenfeld M, Brzezinski A, Levy M, Eliakim M. Fertility and obstetric history in patients with familial Mediterranean fever on longterm colchicine therapy. Br J Obstet Gynaecol 1987 Dec; 94 (12):118691.

17. Ben-Chetrit E, Levy M. Colchicine prophylaxis in familial Mediterranean fever: reappraisal after 15 years. Semin Arthritis Rheum $1991 \mathrm{Feb} ; 20$ (4):241-6.

18. Berkenstadt M, Weisz B, Cuckle H, Di-Castro M, Guetta E, Barkai $G$. Chromosomal abnormalities and birth defects among couples with colchicine treated familial Mediterranean fever. Am J Obstet Gynecol 2005 Oct ;193 (4):1513-6.

19. Venhoff N,Voll RE, Glaser C, Thiel J IL-1-blockade with Anakinra during pregnancy: Retrospective analysis of efficacy and safety in female patients with familial Mediterranean fever Z Rheumatol 2017 Jul ; Epub ahead of print

20. Ilgen U, Küçükşahin O Anakinra use during pregnancy: Report of a case with Familial Mediterranean Fever and infertility. Eur $J$ Rheumatol 2017 Mar ; Epub ahead of print. 